\title{
Comparación de métodos empíricos para la estimación de la evapotranspiración de referencia en Holguín, Cuba
}

\author{
Comparison of empiric methods for the estimation \\ of the reference evapotranspiration on Holguín, Cuba
Juan Alejandro Villazón Gómez 1*, Pavel Noris Noris², Ranses J. Vázquez, Montenegro ${ }^{3}$, George Martín Gutiérrez y Yakelín Cobo Vidal ${ }^{4}$

\section{RESUMEN}

El trabajo se desarrolló con el objetivo de comparar los valores de evapotranspiración obtenidos a partir de cuatro métodos indirectos empíricos en cuatro localidades de interés agrícola en la provincia de Holguín. Fueron utilizados datos medios mensuales de las estaciones meteorológicas de La Jíquima, Guaro, Pinares de Mayarí y Velasco. Los métodos aplicados fueron los de Hargreaves, Hargreaves y Samani, Thornthwaite y Blaney-Criddle, además del FAO Penman-Monteith como patrón. Para comprobar la bondad de ajuste se utilizaron los índices estadísticos desviación media, raíz cuadrática de la desviación media, índice de eficiencia, índice de concordancia, índice de confianza y porcentaje de desviación. Los modelos de Hargreaves y Hargreaves y Samani resultaron los de mejor ajuste con el método patrón en todas las localidades. En Pinares de Mayarí los métodos utilizados resultan poco apropiados para estimar la evapotranspiración.

Palabras clave: Evapotranspiración de referencia, Métodos indirectos, Método de FAO Penman-Monteith.

\begin{abstract}
Evapotranspiration is the main process of soil water loss. It can be estimated from empirical equations, such as the FAO PenmanMonteith. Which, although it is recognized as a standar, requires a great variety of data not always available, which makes the use of other equations easier to use. The work was developed with the objective of comparing the evapotranspiration values obtained from four empirical indirect methods in four locations of agricultural interest in the province of Holguin. Average monthly data from the meteorological stations of La Jíquima, Guaro, Pinares de Mayari and Velasco were used. The methods applied were those of Hargreaves, Hargreaves and Samani, Thornthwaite and Blaney-Criddle, in addition to the FAO Penman-Monteith as standar. To verify the goodness of fit, the statistical indices were used: mean deviation, quadratic root of the mean deviation, efficiency index, concordance index, confidence index and percentage of deviation. The Hargreaves and Hargreaves and Samani models were the best fit with the standard method in all locations. In Pinares de Mayari the methods used are not very suitable for estimating evapotranspiration.
\end{abstract}

Keywords: Reference evapotranspiración, Indirect methods, FAO Penman-Monteith method.

\section{Introducción}

La evapotranspiración (ET) es la pérdida de agua por la combinación de dos procesos separados. En uno el agua se pierde desde la superficie del suelo por evaporación y en el otro a través de la transpiración del cultivo. Ambos procesos ocurren de forma simultánea y resulta difícil distinguir la separación entre ellos. Durante las primeras fases de un cultivo, casi toda el agua se evapora directamente desde el suelo, pero en la medida en que se incrementa la cobertura vegetal la transpiración pasa a ser el

\footnotetext{
1 Universidad de Holguín. Sede José de la Luz y Caballero, Facultad de Ciencias Agropecuarias, Centro de Estudios para Agroecosistemas Áridos (CEAAR).Holguín, Cuba.

2 Centro Provincial de Meteorología, Delegación Provincial del Ministerio de Ciencias, Tecnología y Medio Ambiente (CITMA), Holguín, Cuba.

3 Instituto de Meteorología, Ministerio de Ciencias, Tecnología y Medio Ambiente (CITMA), La Habana, Cuba.

4 Estación Provincial de Investigaciones de la Caña de Azúcar (EPICA), Subdirección de I+D, Holguín, Cuba.

* Autor por correspondencia: villazon@uho.edu.cu.
} 
proceso más importante. Los principales factores climáticos que afectan a la evapotranspiración son la radiación solar, la temperatura del aire, la humedad atmosférica y la velocidad del viento (Allen et al., 2006).

La máxima cantidad de agua que pasa a la atmósfera desde una superficie de suelo totalmente cubierto por la vegetación, en circunstancias óptimas y sin déficit hídrico (en dependencia solamente de las condiciones climáticas), se conoce como evapotranspiración potencial (ETP). La que se hace más precisa si se maneja el concepto de evapotranspiración del cultivo de referencia (ETo), que actualmente ha sido sustituido por el de evapotranspiración de referencia (Sánchez y Carvacho, 2011).

La ETo se refiere a la influencia de las condiciones climáticas sobre la evapotranspiración que ocurre desde una superficie de referencia cubierta con un cultivo hipotético de pastos bajo determinadas condiciones (Allen et al., 2006). Si bien los conceptos de evapotranspiración potencial y evapotranspiración de referencia son diferentes en el sentido estricto, ambos indican cantidades máximas de agua perdidas hacia la atmósfera, por lo que en determinadas condiciones pueden considerarse similares (Sánchez y Carvacho, 2011). De esta manera, algunos autores (Bautista et al., 2009; Vega y Jara, 2009) consideran que para este tipo de estudio tal distinción es innecesaria.

Según Sánchez (2001), los métodos para determinar la evapotranspiración se pueden clasificar en dos grandes grupos. Por un lado, tenemos a los métodos de medición o directos, y por otro, a los métodos de estimación o indirectos. A través de los métodos de medición o directos se obtienen los valores más precisos y confiables, pero resulta muy complicada la determinación de la evapotranspiración debido a los instrumentos necesarios para su realización. Por ello, los métodos de estimación o indirectos, que solo requieren datos proporcionados por estaciones meteorológicas, se encuentran más difundidos y se utilizan más a la hora de realizar estudios en amplias extensiones de tierra (Sánchez, 2001).

Dentro de los métodos indirectos se hallan los empíricos, que relacionan la evapotranspiración con variables climáticas. Existen métodos basados en la temperatura del aire y datos astronómicos (Thornthwaite y Blaney-Criddle), en la temperatura del aire y la humedad relativa (Hargreaves), en la temperatura del aire y la radiación solar y métodos basados en la ecuación de combinación del balance de energía y de la transferencia turbulenta del vapor de agua (Penman-Monteith) (Sánchez, 2001).

Si bien los valores de evapotranspiración obtenidos a partir del método de FAO PenmanMonteith son considerados como patrón (Martins et al., 2014) y presentan la posibilidad de usarlo sin restricción en todo tipo de clima, con un alto nivel de ajuste, esta ecuación presenta la desventaja de demandar una gran cantidad de datos que no siempre se encuentran disponibles (Sánchez, 2001).

El objetivo del presente trabajo es comparar los valores de evapotranspiración obtenidos a partir de cuatro métodos indirectos empíricos en cuatro localidades de interés agrícola en la provincia de Holguín.

\section{Materiales y métodos}

El trabajo se desarrolló con los datos tomados, en el período 2006-2015, en cuatro estaciones meteorológicas de la provincia de Holguín (Tabla 1). Se revisaron las bases de datos de las estaciones meteorológicas para encontrar datos faltantes y se realizó un análisis de correlación entre estaciones cercanas o similares en cuanto a características geográficas para completar los datos faltantes mediante regresión.

Se utilizaron métodos empíricos que relacionan la evapotranspiración con las variables climáticas perteneciente a los dos primeros grupos mencionados por Sánchez (2001).

\section{Ecuación de Hargreaves:}

$$
\mathrm{Et}_{0}=0,0135 *\left(\mathrm{t}_{\text {med }}+17,78\right) * \mathrm{R}_{\mathrm{s}}
$$

En la que $E T_{0}$ es la evapotranspiración de referencia, $t_{\text {med }}$ es la temperatura media diaria $\left({ }^{\circ} \mathrm{C}\right)$, $R s$ es la radiación solar incidente diaria, que se calcula por la ecuación de Samani (2000):

$$
\mathrm{R}_{\mathrm{s}}=\mathrm{R}_{0} * \mathrm{KT} *\left(\mathrm{t}_{\max }-\mathrm{t}_{\mathrm{mín}}\right)^{0,5}
$$

En la que $R_{0}$ es la radiación solar extraterrestre (tabulada); $K T$ es un coeficiente igual a 0,162 para regiones interiores y de 0,19 para regiones costeras, según Allen et al. (2006). De la misma forma, $t_{\text {máx }}$ es la temperatura diaria máxima y $t_{\text {min }}$ es la temperatura mínima diaria. 
Tabla 1. Ubicación geográfica y características climáticas de las estaciones meteorológicas.

\begin{tabular}{|c|c|c|c|c|c|}
\hline \multirow{2}{*}{$\begin{array}{c}\text { Estaciones } \\
\text { meteorológicas }\end{array}$} & \multicolumn{2}{|c|}{ Coordenadas } & \multirow{2}{*}{$\begin{array}{l}\text { Precipitación } \\
\text { anual (mm) }\end{array}$} & \multirow{2}{*}{$\begin{array}{c}\text { Temperatura } \\
\text { media }\left({ }^{\circ} \mathrm{C}\right)\end{array}$} & \multirow{2}{*}{$\begin{array}{c}\text { Altitud } \\
(\mathrm{msnmm})\end{array}$} \\
\hline & Latitud N & $\overline{\text { Longitud W }}$ & & & \\
\hline La Jíquima & $20^{\circ} 59^{\prime} 18^{\prime \prime}$ & $76^{\circ} 32^{\prime} 23^{\prime \prime}$ & 1189,8 & 25,4 & 106,77 \\
\hline Guaro & $20^{\circ} 40^{\prime} 21^{\prime \prime}$ & $75^{\circ} 46^{\prime} 57^{\prime \prime}$ & 1099,3 & 25,6 & 20,96 \\
\hline Pinares de Mayarí & $20^{\circ} 29^{\prime} 14^{\prime \prime}$ & $75^{\circ} 47^{\prime} 26^{\prime \prime}$ & 1871,0 & 21,6 & 647,04 \\
\hline Velasco & $21^{\circ} 04^{\prime} 03^{\prime \prime}$ & $76^{\circ} 20^{\prime} 42^{\prime \prime}$ & 1197,2 & 25,7 & 61,00 \\
\hline
\end{tabular}

msnmm: metros sobre el nivel medio del mar.

\section{Ecuación de Hargreaves y Samani:}

$$
\begin{gathered}
\mathrm{ET}_{0}=0,0023 *\left(\mathrm{t}_{\text {med }}+17,78\right) * \\
\mathrm{R}_{0} * \mathrm{R}_{0} *\left(\mathrm{t}_{\text {máx }}-\mathrm{t}_{\text {mín }}\right)^{0,5}
\end{gathered}
$$

Donde $E T_{0}$ es la evapotranspiración de referencia, $t_{\text {med }}$ es la temperatura media diaria $\left({ }^{\circ} \mathrm{C}\right)$, $R_{0}$ es la radiación solar extraterrestre (tabulada, en $\mathrm{mm}$ día $\left.{ }^{-1}\right), t_{\text {máx }}$ es la temperatura máxima diaria $\left({ }^{\circ} \mathrm{C}\right)$ y $t_{\min }$ es la temperatura mínima diaria $\left({ }^{\circ} \mathrm{C}\right)$.

\section{Ecuación de Thornthwaite:}

$$
\mathrm{ETP}=1,6 *\left(\frac{10 * \mathrm{~T}}{\mathrm{I}}\right)^{\mathrm{a}}
$$

Donde ETP es la evapotranspiración potencial (no ajustada) en $\mathrm{cm}$, para meses de 30 días y 12 horas de luz; $T$ es la temperatura media mensual $\left({ }^{\circ} \mathrm{C}\right)$ e $I$ es el índice anual de calor:

$$
I=\sum_{i=1}^{i=12} i
$$

En la que $i$ es el índice de calor mensual, que se calculó:

$$
\mathrm{i}=\left(\frac{\mathrm{T}}{5}\right)^{1,514}
$$

Donde $T$ es la temperatura media mensual.

También en (4):

$$
\begin{gathered}
a=0,000000675 * I^{3}-0,0000771 * \\
I^{2}+0,017925 * I+0,49239
\end{gathered}
$$

La ETP de Thornthwaite obtenida a partir de (1) se ajustó a la duración máxima posible de la insolación para el mes y la latitud en cuestión, mediante el factor obtenido a partir de la expresión:

$$
\mathrm{f}=\frac{\mathrm{N}}{12}
$$

en la que $N$ es un coeficiente tabulado.

Finalmente:

$$
\mathrm{ETP}_{\text {ajustada }}=\mathrm{ETP}_{\text {sin ajustar }} * \mathrm{f}
$$

para meses de 30 días, y:

$$
\mathrm{ETP}_{\text {media diaria }}=\frac{\mathrm{ETP}_{\text {ajustada }}}{30}
$$

El resultado de (10) se multiplicó por la cantidad real de días mensuales.

\section{Ecuación de Blaney-Criddle:}

$$
\mathrm{ETP}=\frac{\mathrm{Ii}}{\mathrm{I}} *\left(45,72 * \mathrm{t}_{\mathrm{mi}}+812,8\right)
$$

El cociente $I_{i} / I$ fue sustituido por el parámetro $p_{i}$, que representa el porcentaje mensual de horas de luz con relación al año (tabulado) por latitud. De esta forma:

$$
\mathrm{ETP}=\rho \mathrm{i} *\left(45,72 * \mathrm{t}_{\mathrm{mi}}+812,8\right)
$$

Los métodos empíricos fueron validados a partir de la ecuación de FAO Penman-Monteith, recomendada por la FAO. Este modelo estima la evapotranspiración con bastante precisión y en las más variadas condiciones climáticas (Allen et al., 2006).

\section{Ecuación de FAO Penman-Monteith:}

$$
\mathrm{ET}_{\mathrm{o}}=\frac{0,408 \Delta\left(\mathrm{R}_{\mathrm{n}}-\mathrm{G}\right)+\gamma \frac{900}{\mathrm{~T}+273} \mathrm{u}_{2}\left(\mathrm{e}_{\mathrm{s}}-\mathrm{e}_{\mathrm{a}}\right)}{\Delta+\gamma\left(1+0,34 \mathrm{u}_{2}\right)}
$$


Donde ETo es la evapotranspiración de referencia (mm día $\left.{ }^{-1}\right), \Delta$ es la pendiente de la curva de presión de vapor $\left(\mathrm{kPa}^{\circ} \mathrm{C}^{-1}\right), R n$ es la radiación neta en la superficie del cultivo ( $\mathrm{MJ} \mathrm{m}^{-2}$ día $^{-1}$ ), $G$ es el flujo de calor del suelo, $\gamma$ es la constante psicrométrica $\left(\mathrm{kPa}^{\circ} \mathrm{C}^{-1}\right), T$ es la temperatura media del aire a $2 \mathrm{~m}$ de altura $\left({ }^{\circ} \mathrm{C}^{-1}\right)$ y $u_{2}$ es la velocidad del viento a $2 \mathrm{~m}$ de altura $\left(\mathrm{m} \mathrm{s}^{-2}\right)$.

El desempeño de los métodos fue validado a partir del coeficiente de regresión, mediante la utilización del software STATISTICA 8, y los siguientes indicadores estadísticos.

Desviación media (Mean deviation, $M D$ ):

$$
\mathrm{MD}=\frac{1}{\mathrm{n}} \sum_{\mathrm{i}=1}^{\mathrm{n}} \mathrm{ETi}_{\mathrm{e}}-\mathrm{ETi}_{\mathrm{m}}
$$

Donde $n$ es el número de pares de datos, $E T i_{e}$ es la i-enésima observación de la evapotranspiración estimada por el modelo a comparar y $E T i_{m}$ es la i-enésima observación de la evapotranspiración estimada por el método FAO Penman-Monteith. La desviación media $(M D)$ puede oscilar en un rango de $-\infty$ a $+\infty$. Cuando la desviación media se encuentra por debajo de cero los valores observados se encuentran sobreestimados y por encima de cero, dichos valores fueron subestimados. El valor óptimo de $M D$ es cero; mientras más baja sea la magnitud de sus valores, mayor será la exactitud de la estimación realizada.

Raíz cuadrática de la desviación media (Root mean square deviation, $\boldsymbol{R M S D}$ ):

$$
\mathrm{RMSD}=\sqrt{\frac{1}{\mathrm{n}} \sum_{\mathrm{i}=1}^{\mathrm{n}}\left(\mathrm{ETi}_{\mathrm{e}}-\mathrm{ETi}_{\mathrm{m}}\right)^{2}}
$$

Donde $n$ es el número de pares de datos, $E T i_{e}$ es la i-enésima observación de la evapotranspiración estimada por el modelo a comparar yETi $i_{m}$ es la i-enésima observación de la evapotranspiración estimada por el método de FAO Penman-Monteith.

Índice de Eficiencia Nash-Sutcliffe (NashSutcliffe Efficiency Index, $E F$ ):

$$
\mathrm{EF}=1-\left|\frac{\sum_{\mathrm{i}=1}^{\mathrm{n}}\left(\mathrm{ETi}_{\mathrm{e}}-\mathrm{ETi}_{\mathrm{m}}\right)^{2}}{\sum_{\mathrm{i}=1}^{\mathrm{n}}\left(\mathrm{ETi}_{\mathrm{m}}-\mathrm{ETmean}_{\mathrm{m}}\right)^{2}}\right|
$$

Donde $n$ es el número de pares de datos, $E T i_{e}$ es la i-enésima observación de la evapotranspiración estimada por el modelo a comparar, $E T i_{m}$ es la i-enésima observación de la evapotranspiración estimada por el método de FAO Penman-Monteith y ETmean $_{m}$ es la media de la evapotranspiración estimada por el método de FAO Penman-Monteith. Determina la magnitud relativa de la varianza residual con la varianza de los datos observados. Esta magnitud tiene un valor óptimo igual a 1,0, los valores entre 0 y 1 generalmente se consideran aceptables.

Índice de concordancia (Index of agreement, $d$ ) (Wilmott, 1985):

$$
\mathrm{d}=1\left[\frac{\sum_{\mathrm{i}=1}^{\mathrm{n}}\left(\mathrm{ETi}_{\mathrm{e}}-\mathrm{ETi}_{\mathrm{m}}\right)^{2}}{\sum_{\mathrm{i}=1}^{\mathrm{n}}\left(\left|\mathrm{ETi}_{\mathrm{e}}-\mathrm{ETmean}_{\mathrm{m}}\right|+\left|\mathrm{ETi}_{\mathrm{m}}-\mathrm{ETmean}_{\mathrm{m}}\right|\right)^{2}}\right]
$$

Donde $n$ es el número de pares de datos, $E T i_{e}$ es la i-enésima observación de la evapotranspiración estimada por el modelo a comparar, $E T i_{m}$ es la i-enésima observación de la evapotranspiración estimada por el método de FAO Penman-Monteith y ETmean $_{m}$ es la media de la evapotranspiración estimada por el método de FAO Penman-Monteith. Es una medida estandarizada del grado de error de la predicción del modelo, en un rango desde 0 hasta 1. Cuando $d=1$, existe una concordancia perfecta entre los valores determinados experimentalmente y los estimados por el modelo; en sentido contrario, cuando $d=0$, nos indica falta de concordancia entre las variables.

Coeficiente de confianza o desempeño $(c)$ (Camargo y Sentelhas, 1997):

$$
\mathrm{c}=\mathrm{d} * \mathrm{r}
$$

Donde $d$ es el índice de concordancia y $r$ es el coeficiente de correlación. Los valores de $c$ fueron clasificados de acuerdo a las categorías propuestas por Camargo y Sentelhas (1997).

\section{Porcentaje de variación}

Para el cálculo de la sobreestimación o subestimación con respecto a la ET de FAO Penman-Monteith:

$$
\mathrm{PV}(\%)=\left(\frac{\mathrm{ET}_{\mathrm{em}} * 100}{\mathrm{ET}_{\mathrm{FAO} \text { Penman-Monteith }}}\right)
$$

Donde $P V$ es el porcentaje de variación (\%), $E T_{e m}$ es la evapotranspiración estimada con los 
modelos en estudio y $E T_{F A O}$ Penman-Monteith es la evapotranspiración determinada por el método de FAO Penman-Monteith.

\section{Resultados y discusión}

La Figura 1 muestra los modelos de regresión entre la ET de FAO Penman-Monteith la ET estimada a partir de las ecuaciones de Hargreaves, Hargreaves y Samani, Thornthwaite y Blaney-Criddle en cuatro localidades de la provincia de Holguín. Puede apreciarse que los modelos de Hargreaves y de Hargreaves y Samani son los de mejor ajuste, al encontrarse el coeficiente de determinación $\left(r^{2}\right)$ más cercano a 1. Los métodos de peor ajuste del coeficiente de determinación son los que incorporan la temperatura del aire y datos astronómicos a sus ecuaciones de estimación. Silva et al. (2016), al encontrar un alto valor del coeficiente de determinación $\left(r^{2}=0,90\right)$ entre las estimaciones de la evapotranspiración diaria obtenidas por los métodos de FAO Penman-Monteith y Hargreaves y Samani, determinaron que en ausencia de los datos climáticos necesarios para calcular la ETo por el método patrón, puede utilizarse la ecuación de Hargreaves y Samani para determinar la Eto en la región de Arapiraca-AL, Brasil.

Bandeira (2016), al utilizar varios métodos para estimar la evapotranspiración de referencia en la región central del estado de Ceará (Brasil) y compararlos con las estimaciones obtenidas por FAO Penman-Monteith, encontró valores del coeficiente de determinación de 0,8152 y 0,7916 para los métodos de Blaney-Criddle y Hargreaves y Samani, respectivamente. También Cunha et al. (2017a), al comparar la ecuación de FAO Penman-Monteith con los métodos de Hargreaves y Samani y BlaneyCriddle para la estimación de la evapotranspiración de referencia diaria, encontraron ajustes óptimos del coeficiente de determinación.

El peor comportamiento por localidades se encontró en Pinares de Mayarí donde, comparados con la evapotranspiración obtenida en el resto de las estaciones meteorológicas, las funciones de regresión determinadas muestran una dispersión de los datos más abierta.

La Tabla 2 muestra la comparación de los valores de $E T$ obtenidos por los diferentes métodos con la ETo de FAO Penman-Monteith. En cuanto a la desviación media y a la raíz cuadrática de la desviación media la estación meteorológica de Pinares de Mayarí fue la de peor comportamiento.

El índice de eficiencia Nash-Sutcliffe tuvo un mejor comportamiento en Guaro y La Jíquima, con el método de Hargreaves y en la primera localidad mencionada con el método de Hargreaves y Samani. Al determinar valores negativos para las ecuaciones
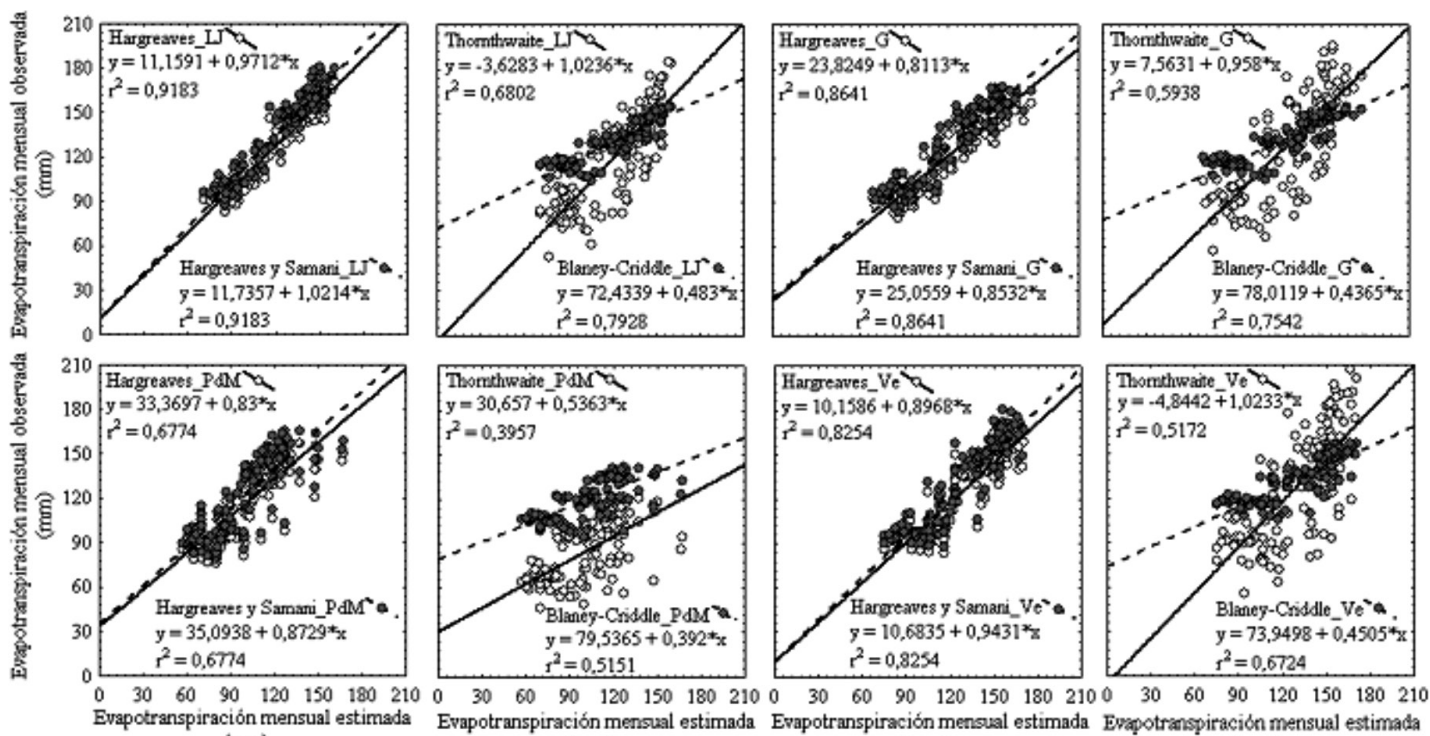

Figura 1. Ecuaciones de regresión a partir de la evapotranspiración estimada por el método de FAO Penman-Monteith (regresora) y la ET estimada por los modelos (dependientes). 
Tabla 2. Validación de los métodos comparados con el método de FAO Penman-Monteith.

\begin{tabular}{|c|c|c|c|c|}
\hline Estaciones meteorológicas & Hargreaves & Hargreaves y Samani & Thornthwaite & Blaney-Criddle \\
\hline \multicolumn{5}{|c|}{ Desviación media } \\
\hline La Jíquima & $-7,661$ & $-14,342$ & 0,758 & $-9,550$ \\
\hline Guaro & $-0,302$ & $-6,759$ & $-2,321$ & $-7,761$ \\
\hline Pinares de Mayarí & $-15,724$ & $-21,900$ & 17,476 & $-16,420$ \\
\hline Velasco & 3,302 & $-3,265$ & 1,809 & $-2,290$ \\
\hline \multicolumn{5}{|c|}{ Raíz cuadrática de la desviación media } \\
\hline La Jíquima & 10,788 & 16,407 & 1,046 & 17,739 \\
\hline Guaro & 10,621 & 12,486 & 3,268 & 19,175 \\
\hline Pinares de Mayarí & 21,360 & 26,486 & 26,239 & 23,892 \\
\hline Velasco & 11,820 & 12,126 & 1,656 & 17,057 \\
\hline \multicolumn{5}{|c|}{ Índice de eficiencia Nash-Sutcliffe } \\
\hline La Jíquima & 0,829 & 0,605 & 0,506 & 0,538 \\
\hline Guaro & 0,861 & 0,808 & 0,364 & 0,546 \\
\hline Pinares de Mayarí & 0,221 & $-0,198$ & $-0,176$ & 0,025 \\
\hline Velasco & 0,804 & 0,794 & 0,017 & 0,592 \\
\hline \multicolumn{5}{|c|}{ Índice de concordancia } \\
\hline La Jíquima & 0,958 & 0,912 & 0,892 & 0,807 \\
\hline Guaro & 0,959 & 0,947 & 0,858 & 0,794 \\
\hline Pinares de Mayarí & 0,810 & 0,745 & 0,646 & 0,617 \\
\hline Velasco & 0,948 & 0,948 & 0,806 & 0,815 \\
\hline \multicolumn{5}{|c|}{ Coeficiente de confianza } \\
\hline La Jíquima & 0,918 & 0,874 & 0,736 & 0,719 \\
\hline Guaro & 0,891 & 0,880 & 0,661 & 0,689 \\
\hline Pinares de Mayarí & 0,667 & 0,613 & 0,406 & 0,443 \\
\hline Velasco & 0,861 & 0,861 & 0,580 & 0,669 \\
\hline \multicolumn{5}{|c|}{ Porcentaje de variación (\%) } \\
\hline La Jíquima & 6,84 & $-10,59$ & 3,46 & $-8,13$ \\
\hline Guaro & 1,45 & $-5,43$ & 1,42 & $-6,79$ \\
\hline Pinares de Mayarí & 16,98 & $-17,15$ & 23,36 & $-14,20$ \\
\hline Velasco & $-2,04$ & $-1,98$ & 6,45 & $-2,36$ \\
\hline
\end{tabular}

de Hargreaves y Samani y Thornthwaite en Pinares de Mayarí, se considera que, en dicha localidad, estos métodos no estiman la evapotranspiración de forma eficiente.

Con respecto al índice de concordancia, en todas las localidades la ecuación de Hargreaves, seguida por la de Hargreaves y Samani, alcanzó los valores más cercanos a 1; lo que indica una concordancia cercana a la perfección. De todas las localidades, la de Pinares de Mayarí fue la de resultados más pobres.

Los resultados obtenidos con el coeficiente de confianza se comportaron de forma similar a los índices de eficiencia y de concordancia. Nuevamente, las ecuaciones de Hargreaves y Hargreaves y
Samani fueron las de mejor comportamiento en las cuatro localidades y Pinares de Mayarí fue la estación meteorológica con el coeficiente de confianza más bajo.

Rodrigues et al. (2017), al analizar en la ciudad de Poço Verde en el estado de Sergipe (Brasil) el comportamiento de la evapotranspiración estimada a partir de varios modelos, encontraron que el coeficiente de confianza obtenido a partir del índice de concordancia y el coeficiente de correlación entre los valores de evapotranspiración por el método de FAO Penman-Monteith (patrón) y por el de Hargreaves y Samani y el de Blaney-Criddle eran tolerable e intermedio, respectivamente. 
En el porcentaje de variación puede observarse que las ecuaciones de Hargreaves (excepto en la localidad de Velasco) y Thornthwaite, tienden a sobreestimar la ETo de FAO Penman-Monteih, mientras que las ecuaciones de Hargreaves y Samani y de Blaney-Criddle subestiman los valores de la evapotranspiración.

Estos resultados coinciden con los encontrados por Cunha et al. (2017a) y Silva et al. (2016) quienes, a pesar de los altos coeficientes de determinación, encontraron que la ecuación de Hargreaves y Samani subestimaba los valores diarios de evapotranspiración obtenidos por la ecuación de FAO Penman-Monteith. También Cunha et al. (2017b) encontraron que las ecuaciones de Hargreaves y Samani y de BlaneyCriddle subestimaban los valores diarios de evapotranspiración de la ecuación patrón en un 27,54 y un $29,85 \%$, respectivamente.

\section{Conclusiones}

Los modelos de Hargreaves y Hargreaves y Samani, sobre todo el primero, tienden a ser los más consistentes con respecto al método de FAO Penman-Monteith para la estimación de la evapotranspiración obtenida por los cuatro métodos, en todas las localidades. Por otro lado, las ecuaciones de Thornthwaite y de Blaney-Criddle presentan una mayor dispersión y un peor ajuste con FAO Penman-Monteith. En la localidad de Pinares de Mayarí, los métodos se muestran poco adecuados para la estimación de la evapotranspiración.

Se confirma que las ecuaciones de Hargreaves constituyen una buena alternativa ante la imposibilidad del cálculo de ecuaciones de mayor demanda de datos; si se considera, además, que la temperatura es una de las variables de registro más común y que puede ser interpolada, espacial y temporalmente, con mayor seguridad que cualquier otra variable meteorológica.

\section{Literatura Citada}

Allen, R.G.; Doorenbos, J.; Monteith, L.

2006. Evapotranspiración del cultivo. Guía para la determinación de los requerimientos de agua de los cultivos. Estudio FAO Riego y Drenaje, boletín 56. Roma. 298 p.

Bandeira da Silva, E.

2016. Estimativa da evapotranspiração por diferentes métodos para a cidade de Quixeramobim, Ceará. Revista Brasileira de Agricultura Irrigada, 10(5): 935-942.

Bautista, F.; Bautista, D.; Delgado Carrazana, C. 2009. Calibration of the equations of Hargreaves and Thornthwaite to estimate the potential evapotranspiration in semi-arid andsubhumid tropical climates for regional applications. Atmósfera, 22(4): 333-348.

Camargo, A.P.; Sentelhas, P.C.

1997. Avaliação do desempenho de diferentes métodos de estimativa da evapotranspiração potencial no Estado de São Paulo. Revista Brasileira de Agrometeorologia, 5: 89-97.

Cunha Barros, A.; Oliveira Aguiar Netto, A. de; Fernandes de Medeiros, P.R.; Monteiro Menezes da Silva, T.; Almeida Diniz Albuquerque, K.

2017b. Comparação entre métodos de estimativa da evapotranspiração de referência em perímetro irrigado no baixo São Francisco. Revista Brasileira de Agricultura Irrigada, 11(8): 2183-2193.

Cunha Barros, A.; Oliveira Silva, C.S. de; Oliveira Aguiar Netto, A. de.

2017a. Ajuste dos parâmetros da equação Hargreaves-Samani em escala diária para o perímetro irrigado Jacaré-Curituba, Canindé-SE. Revista Brasileira de Agricultura Irrigada, 11(8): 2152-2161.

Martins de Souza, J.; Rosa Pereira; L.; Matta Rafael, A. da; Dias da Silva, L.; Fialho dos Reis, E.; Bonomo, R.
2014. Comparison of methods for estimating reference evapotranspiration in two locations of Espirito Santo. Revista Brasileira de Agricultura Irrigada, 8(2): 114-126. Samani, Z.

2000. Estimating solar radiation and evapotranspiration using minimum climatological data. Journal of Irrigation and Drainage Engineering, 126(4): 265-267.

Sánchez Martínez, M.I.

2001. Métodos de estimación de evapotranspiración utilizados en Chile. Revista de Geografía Norte Grande, 28: 3-10.

Sánchez Martínez, M.I.; Carvacho Bart, L.

2011. Comparación de ecuaciones empíricas para el cálculo de la evapotranspiración de referencia en la Región del Libertador General Bernardo O'Higgins, Chile. Revista de Geografía Norte Grande, 50: 171-186.

Silva dos Santos, C.; Pereira dos Santos, D.; Oliveira, W.J. de; Ferreira da Silva, P.; Lins dos Santos, M.A.; Pereira Brito Fontenele, A.J.

2016. Evapotranspiração de referência e coeficiente de cultivo da pimenteira no agreste alagoano. Revista Brasileira de Agricultura Irrigada, 10(5): 883-892.

Rodrigues Gomes Filho, R.; Nascimento Santos, I.L.; Gonzaga de Souza, L.; Vieira Santos, K.; Brito de Oliveira, D.T.; Costa de Jesus, E.

2017. Estimate of the reference evapotranspiration of the Poço Verde region. Revista Brasileira de Agricultura Irrigada, 11(5): 1714-1722.

Vega, E.J.; Jara, J.C.

2009. Estimación dela evapotranspiración de referencia para dos zonas (costa y región andina) del Ecuador. Engenharia Agrícola, 9(3): 390-403.

Willmott, C.J.

1985. On the validation of models. Physical Geography, 2: 84-194. 
